\title{
Weighted Distributions: A Brief Review, Perspective and Characterizations
}

\author{
Aamir Saghir ${ }^{1}$, G. G. Hamedani ${ }^{2}$, Sadaf Tazeem ${ }^{1} \&$ Aneeqa Khadim ${ }^{1}$ \\ ${ }^{1}$ Department of Mathematics, Mirpur University of Science and Technology(MUST), Mirpur-10250 (AJK), Pakistan \\ 2 Department of Mathematics, Statistics and Computer Science, Marquette University, Milwaukee, Wisconsin 53201- \\ 1881, USA
}

Correspondence: G. G. Hamedani, Department of Mathematics, Statistics and Computer Science, Marquette University, Milwaukee, Wisconsin 53201-1881, USA. E-mail: gholamhoss.hamedani@ marquette.edu

Received: February 20, 2017 Accepted: March 15, 2017 Online Published: April 18, 2017

doi:10.5539/ijsp.v6n3p109 URL: https://doi.org/10.5539/ijsp.v6n3p109

\begin{abstract}
The weighted distributions are widely used in many fields such as medicine, ecology and reliability, to name a few, for the development of proper statistical models. Weighted distributions are milestone for efficient modeling of statistical data and prediction when the standard distributions are not appropriate. A good deal of studies related to the weight distributions have been published in the literature. In this article, a brief review of these distributions is carried out. Implications of the differing weight models for future research as well as some possible strategies are discussed. Finally, characterizations of these distributions based on a simple relationship between two truncated moments are presented.
\end{abstract}

Keywords: Length-Biased distribution, Double Weighted distribution, Skewness, MLE, Characterizations.

\section{Introduction}

The theory of weighted distributions provides a collective access for the problems of model specification and data interpretation. It provides a technique for fitting models to the unknown weight functions when samples can be taken both from the original distribution and the developed distribution. Weighted distributions take into account the method of ascertainment, by adjusting the probabilities of the actual occurrence of events to arrive at a specification of the probabilities of those events as observed and recorded. The weighted distributions occur frequently in the studies related to reliability, analysis of family data, Meta analysis and analysis of intervention data, biomedicine, ecology and other areas, for the improvement of proper statistical models.

The concept of weighted distributions was provided by Fisher (1934) and Rao (1965). Fisher (1934) studied how the methods of ascertainment can influence the form of the distribution of recorded observations and then Rao (1965) introduced and formulated it in general terms in connection with modeling statistical data when the usual practice of using standard distributions were found to be unsuitable. Weighted distributions are utilized to modulate the probabilities of the events as observed and transcribed. Patil and Rao (1978) presented some useful concepts. We like to mention here the works of Gupta and Keating (1985), Gupta and Kirmani (1990), Oluyede (1999) and references therein. There are two types of weighted distributions: length biased and size biased distributions.

Many authors have employed the concept of weighted distribution for different purposes: Patil and Rao (1978) examined some general models leading to weighted distributions with weight functions not necessarily bounded by unity and studied length biased (size biased) sampling with applications to wildlife populations and human families. Characteristics of many length biased distributions, preservation stability results and comparisons for weighted and length biased distributions were presented by Khatree (1989). Rao (1965) extended the idea of the methods of ascertainment upon estimation of frequencies and introduced the concept of weighted distributions. However, as far as we have gathered, no study has been carried out that provide a forum for the existing works and future research direction on weighted distributions for applied researchers and practitioners. This, we believe, is a big gap in the literature and the current article is an attempt to fill this gap to some extent.

This article reviews the works that have been carried out so far in the field of weighted distributions and their applications. The rest of the article is summarized as follows: Section 2 briefly reviews the weighted distributions. Section 3 details the review of the works on length biased and weighted length biased distributions. Section 4 explains the works on size biased and double weighted distributions and the related works are taken up in Section 5. Section 6 deals with the characterizations of the weighted distributions. Finally, in Section 7, some concluding remarks are presented. 


\section{Weighted Distributions}

Suppose $X$ is a non-negative continuous random variable with pdf (probability density function) $f(x)$. The pdf of the weighted random variable $X_{w}$ is given by:

$$
f_{w}(x)=\frac{w(x) f(x)}{\mu_{w}}, \quad x>0,
$$

where $w(x)$ is a non-negative weight function and $\mu_{w}=E[W(X)]<\infty$. Note that similar definition can be stated for the discrete random variables.

We shall list below the weighted distributions introduced in the literature in a chronological order.

Castillo and Casany (1998) proposed weighted Poisson distribution by considering the Poisson distribution with parameter $\lambda$ and the weight function $w(k)=(k+\alpha)^{r}$. They developed the weighted Poisson distribution with the probability function:

$$
p_{w}(k ; \lambda, r, \alpha)=\frac{(k+\alpha)^{r} \lambda^{k} e^{-\lambda}}{E_{\lambda}\left[(X+\alpha)^{r}\right] k !}, \quad k=0,1, \ldots
$$

where, $\alpha \geq 0, \lambda>0$ and $r \in \mathbb{R}$ are parameters

They expressed equation (2) as:

$$
p_{w}(k ; \lambda, r, \alpha)=\frac{\exp (k \log \lambda+r \log (k+\alpha))}{k ! C(\lambda, r, \alpha)},
$$

where

$$
C(\lambda, r, \alpha)=e^{\lambda} E_{\lambda}\left[(X+\alpha)^{r}\right]=\sum_{k=0}^{\infty} \frac{(k+\alpha)^{r} \lambda^{k}}{k !} .
$$

The main purpose of their work was to introduce new exponential families that resulted from the concept of the weighted distribution. They derived some statistical properties of the families and provided useful interpretation of the parameters.

Fay et al. (2008) proposed a new metric weighted spectral distribution that improves the graph spectrum by discounting those eigen values that are believed to be unimportant and emphasizing the contributions of those believed to be important. They utilized this metric to optimize the selection of the parameter values of Internet topology generation.

Gupta and Kundu (2009) have followed a similar approach as of Azzalini for introducing a shape parameter to the exponential distribution. They showed that by applying Azzalini's method to the exponential distribution, a new class of weighted exponential distribution can be obtained. The two-parameter weighted exponential distribution (TPWED) introduced by Gupta and Kundu (2009) has pdf:

$$
f_{w}(x ; \alpha, \beta)=\frac{\alpha+1}{\alpha} \beta\left(1-e^{-\alpha \beta x}\right) e^{-\alpha \beta x}, x>0,
$$

where, $\alpha$ and $\beta$ are positive parameters.

Furthermore, they showed that the TPWED possesses some good properties and can be used as a good fit to survival time data compared to other popular distributions such as gamma, Weibull, or generalized exponential distribution.

Kersey (2010) developed the weighted inverse Weibull distribution (WIWD) and beta-inverse Weibull distribution (BIWD). Firstly, taking the weight function $w(x)=x$ and Inverse Weibull pdf:

$$
f(x ; \alpha, \beta)=\beta \alpha^{-\beta} x^{-(\beta+1)} e^{-(\alpha x)^{-\beta}}, x, \alpha, \beta>0,
$$

she developed the WIWD with pdf:

$$
f_{w}(x ; \alpha, \beta)=\frac{\beta \alpha^{1-\beta}}{\Gamma\left(1-\frac{1}{\beta}\right)} x^{-\beta} e^{-(\alpha x)^{-\beta}}, x>0,
$$

where, $\alpha, \beta$ are positive parameters.

Secondly, to develop BIWD, she considered the pdf and cdf (cumulative distribution function) of the beta distribution:

$$
f(x ; a, b)=\frac{1}{B(a, b)} x^{a-1}(1-x)^{b-1}, 0<x<1, a, b>0,
$$


and

$$
F(x ; a, b)=\frac{1}{B(a, b)} \int_{0}^{x} t^{a-1}(1-t)^{b-1} d t, \quad 0 \leq x \leq 1 .
$$

The pdf and cdf of BIWD are given, respectively, by:

$$
f_{w}(x ; \alpha, \beta, a, b)=\frac{\beta \alpha^{-\beta}}{B(a, b)} x^{-(\beta+1)} e^{-a(\alpha x)^{-\beta}}\left(1-e^{-a(\alpha x)^{-\beta}}\right)^{b-1},
$$

$x>0$, and

$$
F_{w}(x ; \alpha, \beta, a, b)=\frac{B_{F(x)}(a, b)}{B(a, b)}, \quad x \geq 0,
$$

where, $\alpha, \beta, a, b$ are all positive parameters and $B_{F(x)}(a, b)=\int_{0}^{F(x)} t^{a-1}(1-t)^{b-1} d t$.

Kersey then discussed some theoretical properties of the inverse Weibull model, weighted inverse Weibull distribution including the hazard function, reverse hazard function, moments, moment generating function, coefficient of variation, coefficient of skewness, coefficient of kurtosis, Fisher information and Shanon entropy. The estimation for the parameters of the length-biased inverse Weibull distribution via maximum likelihood estimation and method of moment estimation techniques were presented, as well as a test for the detection of length-biasedness in the inverse Weibull model. Table 1 shows mean, standard deviation (STD), coefficient of variation (CV), coefficient of skewness (CS) and coefficient of Kurtosis (CK) for some values of the parameters $\alpha$ and $\beta$ for WIWD.

Table 1. Table of Mean, STD, CV, CK of WIWD

\begin{tabular}{ccccccc}
\hline$\alpha$ & $\beta$ & Mean & STD & CV & CS & CK \\
\hline & 3 & 1.9184 & INF & INF & NAN & NAN \\
\cline { 3 - 7 } 1 & 5 & 1.2791 & 0.5188 & 0.4056 & 5.8578 & INF \\
\cline { 3 - 7 } & 8 & 1.1246 & 0.2276 & 0.2023 & 2.5156 & 18.5192 \\
\cline { 3 - 7 } & 10 & 1.0895 & 0.1666 & 0.1529 & 2.0949 & 12.7770 \\
\hline \multirow{3}{*}{2} & 3 & 0.9892 & INF & INF & NAN & NAN \\
\cline { 3 - 7 } & 5 & 0.6396 & 0.2594 & 0.4056 & 5.8578 & INF \\
\cline { 3 - 7 } & 8 & 0.5623 & 0.1138 & 0.2023 & 2.5156 & 18.5192 \\
\cline { 3 - 7 } & 10 & 0.5447 & 0.0833 & 0.1529 & 2.0949 & 12.7770 \\
\hline \multirow{3}{*}{5} & 3 & 0.3957 & INF & INF & NAN & NAN \\
\cline { 3 - 7 } & 5 & 0.2558 & 0.1038 & 0.4056 & 5.8578 & INF \\
\cline { 2 - 7 } & 8 & 0.2249 & 0.0455 & 0.2023 & 2.5156 & 18.5192 \\
\cline { 2 - 7 } & 10 & 0.2179 & 0.0333 & 0.1529 & 2.0949 & 12.7770 \\
\hline
\end{tabular}

Roman (2010) presented theoretical properties and estimation in weighted Weibull and related distributions. Firstly, he considered the Weibull probability density function as:

$$
f(x ; \theta, \beta)=\frac{\beta}{\theta^{\beta}} x^{\beta-1} e^{-\left(\frac{x}{\theta} \beta^{\beta}\right.}, x>0,
$$

where $\theta$ and $\beta$ are positive parameters and applied the weight function $w(t)=t^{c}$ to obtain the weighted Weibull model with pdf as:

$$
f_{w}(x ; \theta, \beta)=\frac{\beta}{\theta^{\beta+c} \Gamma(c)} x^{c+\beta-1} e^{-\left(\frac{x}{\theta}\right)^{\beta}}, x>0 .
$$

Secondly, he considered the Rayleigh pdf as:

$$
f(x ; \sigma)=\frac{x e^{-\frac{1}{2}\left(\frac{x}{\sigma}\right)^{2}}}{\sigma^{2}}, x>0,
$$

where $\sigma>0$ is a parameter and applied the weight function $w(t)=t^{c}$ to obtain the weighted Rayleigh model with pdf as:

$$
f_{w}(x ; \sigma)=\frac{1}{2^{\frac{c}{2}} \sigma^{c+2} \Gamma(c)} x^{c+1} e^{-\frac{1}{2}\left(\frac{x}{\sigma}\right)^{2}}, \quad x>0 .
$$

He derived some theoretical properties of weighted Weibull distribution and properties of the non-weighted Weibull distribution were also reiterated for comparison. 
Shi et al. (2012) constructed the theoretical properties of Weighted Generalized Rayleigh and related distributions. In their paper, a new class of weighted generalization of the Rayleigh distribution (WGRD) was developed by considering the pdf of two parameter generalized Rayleigh distribution (GRD) as:

$$
f(x ; \theta, k)=\frac{k}{\theta^{\frac{1}{k}} \Gamma\left(\frac{1}{k}\right)} e^{-\left(\frac{k^{k}}{\theta}\right)}, x>0,
$$

where $\theta$ and $k$ are positive parameters and the weight function that they utilized $w(x ; m)=x^{m}$, then developed the WGRD with probability density function:

$$
f_{w}(x ; \theta, k, m)=\frac{k x^{m+1}}{\theta^{\frac{m+2}{k}} \Gamma\left(\frac{m+2}{k}\right)} e^{-\left(\frac{x^{k}}{\theta}\right)}, x>0 .
$$

The statistical properties of GRD and WGRD were obtained. Other important properties including entropy measures, Shannon entropy, $\beta$ entropy and generalized entropy for the WGRD were also derived which are measures of the uncertainty in these distributions.

Ye et al. (2012) introduced a weighted generalized beta distribution (WGBD) of the second kind. They used the following pdf of the generalized beta distribution of the second kind;

$$
f(x ; \alpha, \beta, p, q)=\frac{\alpha x^{\alpha p-1}}{\beta^{\alpha p} B(p, q)\left[1+\left(\frac{x}{\beta}\right)^{\alpha}\right]^{p+q}}, \quad x>0,
$$

where $\alpha, \beta, p, q$ are all positive parameters and the weight function $w(x)=x^{k}$ in the derivation of WGBD of second kind. Then, developed the weighted generalized beta distribution of the second kind (WGBD2) as:

$$
f_{w 2}(x ; \alpha, \beta, p, q, k)=\frac{\alpha x^{\alpha p+k-1}}{\beta^{\alpha p+k} B(p+k / \alpha, q-k / \alpha)\left[1+\left(\frac{x}{\beta}\right)^{\alpha}\right]^{p+q}}, x>0,(19)
$$

where, $\alpha, \beta, p, q, k$ are all positive parameters. They derived some statistical properties and introduced some results on the generalized entropy and Renyi entropy of WGBD2.

Aleem at al. (2013) introduced a class of modified weighted Weibull distribution (MWWD) and its properties. They considered the Weibull pdf and its cumulative distribution function as a weight function respectively as:

$$
\begin{aligned}
& f(x ; \beta, \gamma)=\beta \gamma x^{\gamma-1} \gamma e^{-\beta x^{\gamma}}, \quad x>0, \beta, \gamma>0 . \\
& F_{x}(\theta x)=w(t(x)),
\end{aligned}
$$

where

$$
F_{x(\beta \gamma)}(x)=1-e^{-\beta x^{\gamma}} .
$$

They defined the new class of modified weighted distribution via the probability density function given by;

$$
f_{w}(x)=\frac{[1-w(t x)]^{c} f(x)}{E[1-w(t x)]^{c}}, \quad x \in \mathbb{R},
$$

and

$$
f_{x \theta}(x)=\frac{\left[1-F_{X}(\theta x)\right]^{c} f(x)}{E\left[1-F_{X}(\theta x)\right]^{c}}, \quad x>0 .
$$

Finally, they derived the MWWD $(\beta, \gamma, \theta, c)$ with pdf:

$$
f_{w}(x)=\beta \gamma\left(c \theta^{\gamma}+1\right) x^{\gamma-1} e^{-\beta\left(c \theta^{\gamma}+1\right) x^{\gamma}}, \quad x>0,
$$

where, $\beta, \gamma, \theta, c$ are positive parameters. They derived the basic statistical properties of this new distribution. 
Al-Khadim and Hantoosh (2013) presented the even -power weighted distribution by taking the two types of weight functions $w_{1}(x)=x$ and $w_{2}(x)=e^{x}$ and used the normal distribution. After applying the definition of even -power weighted distribution, they developed the two different pdf of even-power weighted normal distribution $\left(\mathrm{EPWND}_{1}\right)$ i.e.,

$$
f_{w_{1}}\left(x ; \mu, \sigma^{2}\right)=\frac{1}{\sqrt{2 \pi} \sigma\left(\sigma^{2}+\mu^{2}\right)} x^{2} e^{\frac{-(x-\mu)^{2}}{2 \sigma^{2}}}, \quad x>0,
$$

and $\left(\mathrm{EPWND}_{2}\right)$

$$
f_{w_{2}}\left(x ; \mu, \sigma^{2}, r\right)=\frac{1}{\sqrt{2 \pi} \sigma e^{2 r}\left(2 r^{2} \sigma^{2}+\mu\right)} x^{2} e^{\frac{2 r x-(x-\mu)^{2}}{2 \sigma^{2}}}, \quad x>0,
$$

where, $\mu \in \mathbb{R}, \sigma>0$ and $r>0$ are parameters. Then, they discussed some of the statistical properties of these two distributions including entropy.

Mahdy (2013) introduced the new class of weighted Weibull distribution and its properties. In his work, a skewness parameter to a Weibull distribution was introduced using an idea of Azzalini, which creates a new class of weighted Weibull distributions. The new distribution having pdf:

$$
f_{w}(x ; \alpha, \beta, \lambda)=\frac{\lambda \beta\left(1+\alpha^{\beta}\right) x^{\beta-1} e^{-\lambda x^{\beta}}\left(1-e^{-\lambda(\alpha x)^{\beta}}\right)}{\alpha^{\beta}}, \quad x>0,
$$

where, $\alpha, \beta, \lambda$ are all positive parameters.

This new distribution has a probability density function with skewness representing a general case of weighted probability density function of the extreme value distribution, the Rayleigh distribution and exponential distribution. Different properties of this new distribution are discussed and the inference of the old parameters and the skewness parameter is studied.

Badmus et al. (2014) presented Lehmann Type II weighted Weibull distribution. They improved on the method used on weighted Weibull model, proposed by Azzalini (1985), using the logit of Beta function by Jones (2004) to have Lehmann Type II weighted Weibull model. The main purpose of their work was to obtain a distribution that is better than both weighted Weibull and Weibull distribution in terms of estimate of their characteristics and their parameters. Some basic properties of the newly proposed distribution including moments and moment generating function, survival rate function, hazard function, asymptotic behaviors and the estimation of the parameters have been studied.

Idowu and Adebayo (2014) proposed exponentiated weighted Weibull distribution (EWWD). This model was established with a view of obtaining a model that is better than both Weibull and weighted Weibull distributions in terms of the estimate of their characteristics and their parameters using the logit of Beta by Jones (2004) and the new class of weighted Weibull distribution proposed by Mahdy (2013) given in equation (28). They derived the exponentiated-weighted Weibull distribution with pdf:

$$
\begin{gathered}
f_{w}(x ; \alpha, \beta, \lambda)=\alpha\left[\frac{\left(1+\alpha^{\beta}\right)\left(1-e^{-\lambda x^{\beta}}\right)+e^{\lambda x^{\beta}\left(1+\alpha^{\beta}\right)}-1}{\alpha^{\beta}}\right]^{\alpha-1} \times \\
{\left[\frac{\lambda \beta\left(1+\alpha^{\beta}\right) x^{\beta-1} e^{-\lambda x^{\beta}}\left(1-e^{\lambda x^{\beta}}\right)}{\alpha^{\beta}} \alpha^{\beta}\right],}
\end{gathered}
$$

$x>0$, where $\alpha, \beta, \lambda$ are positive parameters. They derived the statistical properties of EWWD and the estimation of the parameters have also been studied.

Odubote and Oluyede (2014) constructed a new six-parameter class of distributions called weighted Feller-Pareto distribution (WFPD). They considered the pdf of Feller-Pareto distribution defined as:

$$
f_{F P}(x ; \mu, \theta, \gamma, \alpha, \beta)=\frac{1}{B[\alpha, \beta]}\left(\frac{x-\mu}{\theta}\right)^{\frac{\beta}{\gamma}-1}\left[1+\left(\frac{x-\mu}{\theta}\right)^{\frac{1}{\gamma}}\right]^{-(\alpha+\beta)}, x>\mu,
$$

where, $\theta, \gamma, \alpha, \beta$ all positive $\mu \in \mathbb{R}$ are parameters and weight function is $\left(\frac{y-\mu}{\theta}\right)^{k}$. The resultant WFPD is:

$$
f_{w}(x ; \mu, \theta, \gamma, \alpha, \beta, k)=\frac{\Gamma(\alpha+\beta)}{\Gamma(k \gamma+\beta) \Gamma(\alpha-k \gamma) \theta \gamma}\left(\frac{x-\mu}{\theta}\right)^{k+\frac{\beta}{\gamma}-1}\left[1+\left(\frac{x-\mu}{\theta}\right)^{\frac{1}{\gamma}}\right]^{-(\alpha+\beta)}, x>0,
$$

where $\alpha, \beta, \theta, \gamma$ are all positive $\mu \in \mathbb{R}, k=0,1,2, \ldots, \alpha-k \gamma>0$ are parameters.

This new class of distributions comprise several other Pareto-type distributions such as length-biased (LB) Pareto, weighted Pareto (WP I, II, III, and IV), and Pareto (WP I, II, III, and IV) distributions as special cases. They derived its monotonicity properties, measures of uncertainty including Renyi, Shannon and s-entropies of the WFPD. 
Alqallaf et al. (2015) studied the estimation of two parameter weighted exponential distribution. The main goal of their work was to compare via Monte Carlo simulation, the finite sample properties of the estimates of the parameters of the weighted exponential distribution obtained from five estimation methods: MLE, MOM, L-moments, ordinary leastsquares and weighted least-squares. They used bias and mean-squared error as the criteria for comparison. They analyzed two real data sets for comparing the five estimation methods for the weighted exponential distribution.

Bashir and Rasul (2015) established the weighted Lindley distribution (WLD) . The main objective of their work was to develop WLD by using single parameter Lindley distribution with pdf:

$$
f(x ; \theta)=\frac{\theta^{2}}{\theta+1}(1+x) e^{-\theta x}, \quad x>0,
$$

where $\theta>0$ is a parameter and the weight function is $w(\phi x)=e^{\phi x}$. Applying the definition of weighted distribution, they obtained WLD having the pdf:

$$
f_{w}(x ; \theta, \phi)=\frac{(\theta-\phi)^{2}}{\theta-\phi+1}(1+x) e^{-(\theta-\phi) x}, x,>0,
$$

where $\theta, \phi$ are positive parameters. They also studied the basic properties of the WLD.

Mahdavi (2015) proposed two weighted distributions generated by the exponential distribution. In his work, he presented new classes of weighted distributions by incorporating exponential distribution via Azzalini's method. Resulting weighted models generated by the exponential distribution were the weighted gamma-exponential model (WGD) with pdf:

$$
f_{w}(x ; \lambda, \alpha)=\frac{\lambda^{k} x^{k-1} e^{-\lambda x}\left(1-e^{-\alpha \lambda x}\right)}{\Gamma(k)\left[1-(1+\alpha)^{-k}\right]}, x>0,
$$

and the weighted generalized exponential (WGED) based on the exponential distribution with pdf:

$$
f_{w}(x ; \lambda, \alpha, \beta)=\frac{\beta \lambda e^{-\lambda x}\left(1-e^{-\lambda x}\right)^{\beta-1}\left(1-e^{-\alpha \lambda x}\right)}{1-\beta B(\alpha+1, \beta)}, x>0,
$$

where, $\lambda, \alpha, \beta$ are all positive parameters.

The moment properties of the proposed distributions were studied. Maximum likelihood estimators of the unknown parameters cannot be obtained in explicit forms and they have to be obtained via numerical methods.

Nasiru (2015) presented another weighted Weibull distribution (WWD) from Azzalini's family. He proposed a new WWD by employing

$$
f(x ; \lambda, \alpha, \theta)=K g_{0}(x) G_{0}(\lambda x), \quad x>0,
$$

where, $\alpha, \theta, \lambda$ are all positive parameters, $g_{0}(x)$ is the pdf of Weibull distribution with parameters $\alpha$ and $\theta$ and $G_{0}(\lambda x)$ is its survival function. He derived the new WWD having pdf:

$$
f_{w}(x ; \lambda, \alpha, \theta)=\left(1+\lambda^{\theta}\right) \alpha \theta x^{\theta-1} e^{-\left(\alpha x^{\theta}+\alpha(\lambda)^{\theta}\right)}, x>0 .
$$

Finally, he discussed some mathematical properties of this new model such as Renyi entropy and order statistics. The method of maximum likelihood was employed for estimating the parameters of the distribution.

Rezzoky and Nadhel (2015) proposed a weighted generalized exponential distribution (WGED) with the known shape parameter $\alpha=2$ and scale parameter $\lambda>0$. They considered the pdf of generalized exponential distribution (GED) with pdf:

$$
f(x ; \lambda, \alpha)=\alpha \lambda\left(1-e^{-\lambda x}\right) e^{-\lambda x}, x>0,
$$

where, $\alpha, \lambda$ are positive parameters and $w(x)=x$ is the weight function. Their GWED has pdf:

$$
f_{w}(x ; \lambda)=\frac{4}{3} x \lambda^{2}\left(1-e^{-\lambda x}\right) e^{-\lambda x}, x>0 .
$$

They established some of its mathematical properties and finally they estimated its scale parameter $\lambda$ using the method of moments and maximum likelihood estimators. The probability plots of WGED for $\lambda=1,2$ and 3 are provided here in Figure 1. 


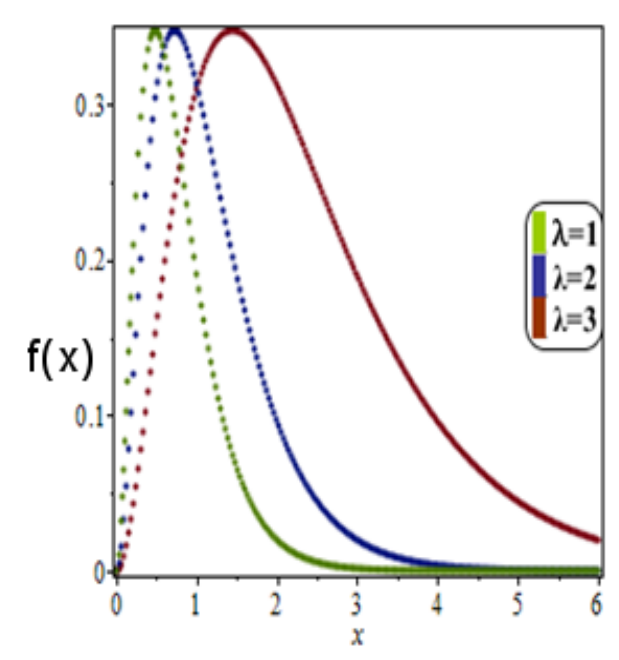

Figure 1 . The probability density function of WGED for different values of $\lambda$

Oguntunde (2015) presented the exponentiated weighted exponential distribution (EWED) and its statistical properties. He discussed the generalization of the weighted exponential distribution (WED) and proposed the pdf of EWED as follows

$$
\begin{aligned}
f_{w}(x ; \alpha, \theta, \lambda) & =\theta\left(\frac{\alpha+1}{\alpha}\right) \lambda e^{-\lambda x} \times \\
& {\left[1-e^{-\lambda \alpha x}\right]\left[\frac{\alpha+1}{\alpha}\left(1-e^{-\lambda x}-\frac{1}{1+\alpha}\left(1-e^{-\lambda x(1+\alpha)}\right)\right)\right]^{\theta-1}, }
\end{aligned}
$$

where, $\alpha, \theta, \lambda$ are positive parameters.

He also studied and provided the basic statistical properties of his proposed model. The method of maximum likelihood estimation was discussed for estimating the parameters of the model.

Asgharzadeh et al. (2016) introduced a new weighted Lindley distribution (NWLD) with application in survival analysis. The probability density function of this distribution is

$$
f_{w}(x ; \lambda, \alpha)=\frac{\lambda^{2}(1+\alpha)^{2}}{\alpha \lambda(1+\alpha)+\alpha(2+\alpha)}(1+x)\left(1-e^{-(\lambda \alpha x)}\right) e^{-(\lambda x)}, \quad x>0,
$$

where, $\alpha, \lambda$ are positive parameters.

The proposed NWLD contains Lindley and weighted Lindley (given by Ghitany et al., 2011) distributions as special cases. Also, the distribution can be represented as a mixture of weighted exponential (Gupta and Kundu, 2009) and weighted gamma distributions; and as a negative mixture of Lindley distributions with different parameters.

\section{Length-Biased and Length-Biased Weighted Distributions}

For the weight function $w(x)=x$ in equation (1), the resultant model is called Length-Biased distribution and its pdf is given by

$$
f_{L}(x)=\frac{x f(x)}{\mu}, \quad x>0 .
$$

If $f(x)$ is a weighted model in (42), the resultant distribution is called length biased weighted distribution (LBWD).

Das and Roy (2011a) developed the length-biased form of the weighted generalized Rayleigh distribution (WGRD) known as length-biased weighted generalized Rayleigh distribution (LBWGRD). Firstly, they considered the general- 
ized Rayleigh pdf:

$$
f\left(x ; N, \sigma^{2}\right)=\frac{2}{\left(2 \sigma^{2}\right)^{\frac{N}{2}} \Gamma\left(\frac{N}{2}\right)} x^{N-1} e^{-\frac{x^{2}}{2 \sigma^{2}}}, \quad x>0,
$$

where, $\sigma>0, N \in \mathbb{N}$ are parameters.

The weight function $w(x)=x^{2 c-N} e^{-x^{2}\left(c \sigma^{2}-\frac{1}{2 \sigma^{2}}\right)}$ was considered in equation (1) to obtain the WGRD. The pdf of WGRD is:

$$
f_{w}\left(x, c, \sigma^{2}\right)=\frac{2\left(c \sigma^{2}\right)^{c} x^{2 c-1} e^{-x^{2} c \sigma^{2}}}{\Gamma(c)}, x>0,
$$

with $c>0$ a parameter as well.

Then, they used the pdf (44) and the weight function $w(x)=x$ to develop the LBWGRD with the following pdf:

$$
f_{w}\left(x, c, \sigma^{2}\right)=\frac{2\left(c \sigma^{2}\right)^{c+1 / 2} x^{2 c} e^{-x^{2} c \sigma^{2}}}{\Gamma(c+1 / 2)}, \quad x>0 .
$$

Then, they discussed some of the properties of LBWGRD. The estimates of the parameters of the LBWGRD were obtained via the method of moments and fitted accordingly.

Das and Roy (2011b)proposed the Length-Biased form of weighted Weibull distribution (WWD). Firstly, they considered the two parameters Weibull distribution with pdf:

$$
f(x, c, \alpha)=\frac{c}{\alpha}\left(\frac{c}{\alpha}\right)^{c-1} e^{-(x / \alpha)^{c}}, \quad x>0,
$$

where, $\alpha, c$ are positive parameters. They used the weight function $w(x)=x^{c \beta}$ and obtained the weighted Weibull distribution with pdf

$$
f_{w}(x, c, \alpha, \beta)=\frac{c}{\alpha^{c+\frac{c}{\beta}} \Gamma(\beta+1)} x^{c+\frac{c}{\beta}-1} e^{-\left(\frac{x}{\alpha}\right)^{c}}, \quad x>0,
$$

where, $\beta>0$ is a parameter as well.

Then, they used the weighted Weibull distribution and the weight function $w(x)=x$, to develop the LBWWD with pdf:

$$
f_{w}(x, c, \alpha, \beta)=\frac{c}{\alpha^{c+c \beta+1} \Gamma(\beta+1+1 / c)} x^{c+c \beta} e^{-\left(\frac{x}{\alpha}\right)^{c}}, \quad x>0 .
$$

They also established various properties of LBWWD. Newby's method along with the method of moment have been used to estimate the parameters of the LBWWD.

Ratnaparkhi and Nimbalkar (2012) developed the length-biased lognormal distribution (LBLND). They considered the log-normal distribution (LND) with pdf

$$
f(x ; \mu, \sigma)=\frac{1}{x \sigma \sqrt{2 \pi}} e^{\frac{-1}{2 \sigma^{2}}(\log x-\mu)^{2}}, \quad x>0,
$$

where $\mu \in \mathbb{R}$ and $\sigma>0$ are parameters. Using the definition of length biased distribution, they obtained the pdf of LBLND given by:

$$
f(x, \mu, \sigma)=\frac{1}{x \sigma \sqrt{2 \pi}} e^{\frac{-1}{\sigma^{2}}\left(\log x-\left(\mu+\sigma^{2}\right)\right)^{2}}, \quad x>0 .
$$

They studied its application in oil field exploration. They considered some related estimation problems and also analyzed the sized-biased data arising in the exploration of oil fields. They discussed some properties of LBLND including mean, median, mode, variance of the estimators using simulations and the use of sample mode as an estimate of the lognormal parameter and discussed briefly the maximum likelihood estimations of the parameters of the LBLND. The following Table 2. presents the different statistical properties of LND and LBLND.

Mir et al. (2013) introduced structural properties of the length-biased beta distribution of first kind $\left(\mathrm{LBBD}_{1}\right)$. In their work, they presented the length-biased form of the weighted beta distribution of first kind (WLBBD $)_{1}$. They presented some structural properties of $\mathrm{WLBBD}_{1}$ by considering pdf of beta distribution of first kind

$$
f(x ; a, b)=\frac{1}{B(a, b)} x^{a-1}(1-x)^{b-1}, \quad 0<x<1,
$$


Table 2. Statistical Properties of LN and LBLN distributions

\begin{tabular}{ccc}
\hline Property & LND & LBND \\
\hline Mean & $\exp \left(\mu+\sigma^{2} / 2\right)$ & $\exp \left(\mu^{*}+\sigma^{2} / 2\right)$ \\
\hline Median & $\exp (\mu)$ & $\exp \left(\mu^{*}\right)$ \\
\hline Mode & $\exp \left(\mu-\sigma^{2}\right)$ & $\exp \left(\mu^{*}-\sigma^{2}\right)$ \\
\hline Variance & $\exp \left(2 \mu+\sigma^{2}\right) \exp \left(\sigma^{2}\right)-1$ & $\exp \left(2 \mu^{*}+\sigma^{2}\right) \exp \left(\sigma^{2}\right)-1$ \\
\hline
\end{tabular}

where, $a, b$ are positive parameters and from (51), the pdf of $\mathrm{WLBBD}_{1}$ is given by:

$$
f_{w}(x, \alpha+1, \beta)=\frac{1}{B(\alpha+1, \beta)} x^{\alpha}(1-x)^{\beta-1}, \quad 0<x<1,
$$

where, $\alpha, \beta$ are positive parameters.

Boudrissa (2013) developed the weighted Weibull length-biased distribution (WWLBD). He considered the Weibull pdf:

$$
f(x ; \beta, \theta)=\beta \theta x^{\beta-1} e^{-\theta x^{\beta}}, \quad x \geq 0, \beta, \theta>0,
$$

and the weight function $w(x)=x$. They obtained the following pdf:

$$
f_{w}(x ; \beta, \theta)=\frac{\beta^{2} \theta^{\left(1+\frac{1}{\beta}\right)} x^{\beta} e^{-\theta x^{\beta}}}{\Gamma\left(\frac{1}{\beta}\right)}, \quad x>0,
$$

where, $\beta, \theta$ are positive parameters.

They derived some properties of WWLBD. They also considered Bayesian and non-Bayesian estimation problems and a numerical example were introduced for illustration using the data from Gupta and Akman (1998), which represent a million of revolutions to failure for 23 ball bearings in fatigue test. These data have been previously fitted assuming Weibull, lognormal, Inverse Gaussian and length-biased inverse Gaussian.

Al-Kadim and Hussein (2014) proposed the length-biased form of weighted exponential and Rayleigh distributions. They considered the exponential and Rayleigh pdf respectively

$$
f(x ; \lambda)=\lambda e^{-\lambda x}, \quad x>0,
$$

and

$$
f(x ; \beta, \theta)=\frac{2 x \beta}{\theta} e^{-\frac{\beta x^{2}}{\theta}}, \quad x>0,
$$

where, $\beta, \theta, \lambda$ are positive parameters.

They utilized the weight functions $w_{1}(x)=e^{n x}$ and $w_{2}(x)=n x / \theta$ respectively for the these distributions to derived their weighted versions. After that they applied the definition of length-biased distribution given in equation (42) and finally obtained LBWED and LBWRD respectively having the pdfs

$$
f_{w_{1}}(x ; \lambda)=x(\lambda-n)^{2} e^{-(\lambda-n) x}, \quad x>0, \lambda>n,
$$

and

$$
f_{w_{2}}(x ; \beta, \theta)=\frac{2 x^{3} \beta^{2}}{\theta^{2}} e^{-\frac{\beta x^{2}}{\theta}}, \quad x, \beta, \theta>0 .
$$

They also studied some of its statistical properties and presented an application of the new distributions.

Nanuwong and Bodhisuwan (2014) presented the length-biased beta-Pareto distribution (LBBPD), its structural properties and its application. They introduced the LBBPD in their article by taking beta-Pareto pdf:

$$
f(x ; \alpha, \beta, \theta, \gamma)=\frac{\gamma}{\theta B(\alpha, \beta)}\left[1-\left(\frac{x}{\theta}\right)^{-} \gamma\right]^{\alpha-1}\left(\frac{x}{\theta}\right)^{\gamma \beta-1}, x \geq 0, \alpha, \beta, \theta, \gamma>0 .
$$

They derived the length biased version of beta-Pareto distribution with pdf:

$$
f_{L}(x ; \alpha, \beta, \theta, \gamma)=\frac{\gamma}{\theta B\left(\alpha, \beta-\frac{1}{\gamma}\right)}\left[1-\left(\frac{x}{\theta}\right)^{-} \gamma\right]^{\alpha-1}\left(\frac{x}{\theta}\right)^{\gamma \beta}, x \geq 0, \alpha, \beta, \theta, \gamma>0 .
$$

They presented some interesting properties of this distribution, such as hazard rate, Renyi and Shannon entropies. They used maximum likelihood estimation to estimate parameters of the distribution. 
Seenoi et al. (2014) proposed the length-biased exponentiated inverted Weibull distribution (LBEIWD) by using the exponentiated inverted Weibull pdf:

$$
f(x ; \beta, \theta)=x^{-(\beta+1)}\left(e^{-x^{-} \beta}\right)^{\theta}, \quad x, \beta, \theta>0,
$$

and weight function $w(x)=x$. Then, they finally developed the LBEIWD with pdf given by:

$$
f(x ; \beta, \theta)=\frac{\beta \theta^{1-1 / \beta}}{\Gamma(1-1 / \beta)} x^{-\beta}\left(e^{-x^{-} \beta}\right)^{\theta}, \quad x, \beta, \theta>0 .
$$

The plots of pdf of LBEIWD are given in Figure 2 below which indicates that for the fixed value of $\beta$, as the value of $\theta$ decreases, the distribution approaches symmetry. Kersey and Oluyede (2015) proposed theoretical properties of the

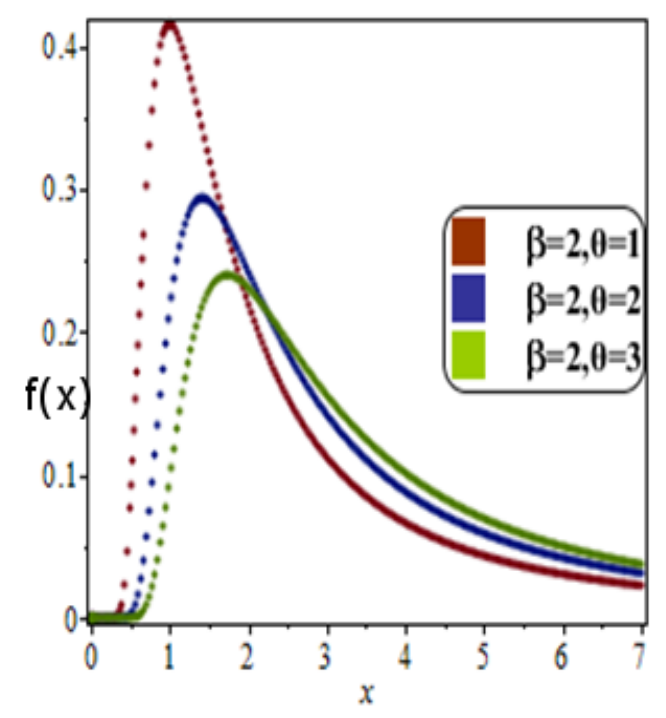

Figure 2. The plots of the pdf of LBEIWD for selected values of parameters

LBIWD. They derived some of its properties including, hazard and reverse hazard functions, reliability function, moments and moment-generating function. Fisher information and Shannon entropy were also presented.

Modi (2015) presented length-biased weighted Maxwell distribution (LBWMD) by using the Maxwell pdf

$$
f(x ; \alpha)=\frac{\sqrt{(2) \alpha^{1} .5 x^{2} e^{-\frac{\alpha x^{2}}{2}}}}{\sqrt{\pi}}, \quad x, \alpha>0,
$$

and the weight function $w(x, t)=e^{\left(t x^{2} / 2\right)}$. Then, he derived the weighted Maxwell distribution of the form:

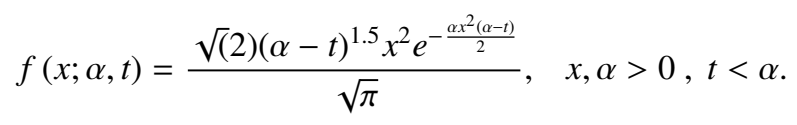

Mudasir and Ahmad (2015) discussed the structural properties of length-biased Nakagami distribution (LBND) by using the Nakagami pdf:

$$
f(x ; \theta, \beta)=\frac{2 \theta^{\theta}}{\Gamma(\theta) \beta^{\theta}} x^{2 \theta-1} e^{-\frac{\theta x^{2}}{\beta}}, \quad x, \beta>0, \theta \geq 0,
$$

and the weight function $w(x)=x$ to obtain LBND with pdf

$$
f_{L}(x ; \theta, \beta)=\frac{2 \theta^{\theta+1 / 2}}{\Gamma \theta+1 / 2 \beta^{\theta+1 / 2}} x^{2 \theta} e^{-\frac{\theta x^{2}}{\beta}}, \quad x, \beta>0, \theta \geq 0 .
$$


Simmachan et al. (2015) proposed a new lifetime distribution based on the re-parameterizations model called two-sided length-biased inverse Gaussian distribution (TSLBIGD). They considered the inverse Gaussian pdf

$$
f(x ; \theta, \lambda)=\frac{\lambda}{\theta \sqrt{2 \pi}}\left(\frac{\theta}{x}\right)^{1.5} e^{-0.5\left(\sqrt{\left.\left.\left(\frac{x}{\theta}\right)-\lambda \sqrt{(} \frac{\theta}{x}\right)\right)^{2}}\right.}, \quad x, \theta, \lambda>0 .
$$

Then, they derived the pdf of TSLBIGD by using the pdf (67) and weight function $w(x)=x$ to obtain:

$$
f_{L}(x ; \theta, \lambda)=\frac{\lambda}{\theta \sqrt{2 \pi}}\left(\frac{\theta}{x}\right)^{0.5} e^{-0.5\left(\sqrt{\left(\lambda \frac{x}{\theta}\right)-\sqrt{\left.\left(\frac{\theta}{x}\right)\right)^{2}}}, \quad x, \theta, \lambda>0 .\right.}
$$

\section{Size - Biased Distribution}

For the weight function $w(x)=x^{c}$ in equation (1), the resultant distribution is named size - biased distribution. The probability density function of the size - biased distribution is given by

$$
f(x)=\frac{x^{c} f(x)}{\mu_{c}}, \quad x>0 .
$$

Size-biased Poisson-Lindley distribution and its application was introduced by Ghitany and Al-Mutairi (2008). In that paper they investigated the method of moments and the maximum likelihood estimators of the parameter of the size-biased Poisson-Lindley distribution. Hassan et al. (2008) introduced misclassified size-biased modified power series distribution and its applications. A misclassified size-biased modified power series distribution where some of the observations corresponding to $x=2$ are misclassified as $x=1$ with probability $\alpha$, was defined. They obtained its recurrence relations among ordinary, central and factorial moments and also for some of its particular cases like the size-biased generalized negative binomial and the size-biased generalized Poisson distributions.

Mir (2009a) presented a new method of estimation of size-biased generalized logarithmic series distribution and obtained its moments. Comparison has been made among different estimation methods by means of Pearson's Chi-square, Akaike Information Criterion (AIC) and Bayesian Information Criterion (BIC) techniques.

Mir (2009b) introduced a size-biased negative binomial distribution and its use in Zero-Truncated Cases. A size-biased negative binomial distribution, a particular case of the weighted negative binomial distribution, taking the weights as the variant values has been defined. A Bayes' estimator of this distribution has been obtained by using non-informative and gamma prior distributions. Also comparison between this estimator with the corresponding maximum likelihood estimator were discussed with the help of R-Software.

Bashir and Ahmad (2014) discussed record values from size-biased Pareto distribution as well as their characterizations. The objective of their paper was to obtain upper record values from the size-biased Pareto distribution and derived distributional properties of this distribution, including pdf, cdf, moments, entropy, inverse/negative moments, relations between negative and positive moments, median, mode, joint and conditional pdfs and conditional mean and variance. The reliability measures of the upper record values from this distribution, such as survival function, hazard rate function, cumulative hazard rate function and reversed hazard rate were also discussed. A characterization of the proposed distribution based on the conditional expectation of record values was given.

Record Values on the size-biased student's t distribution was introduced by Bashir and Akhtar in (2014). Record values have useful applications in many real life data including weather, business, economics and sports. In this paper they discussed the upper record values arising from the size-biased student's t distribution and some applications on real data sets are presented.

Singh and Srivastava (2014) introduced estimation of the parameters in the size-biased inverse Maxwell distribution. The parameters were estimated by the method of maximum likelihood and method of moment. The properties of size-biased inverse Maxwell were discussed and its survival and hazard functions were obtained. These functions were plotted, their properties were investigated and test for this distribution was suggested.

Adhikari and Srivastava (2014) proposed a Poisson-size-biased Lindley distribution and the corresponding pmf obtained. Some of its properties and the expressions for raw and central moments, coefficients of skewness and kurtosis were derived. The moment equations and the maximum likelihood estimators of the parameters of this Poisson size-biased Lindley distribution were obtained.

Jabeen and Jan (2015) provided information measures of size biased generalized gamma distribution such as entropy, entropy estimation, Kullback-Leibler discrimination, data transformation, log-likelihood ratio and Akaike and Bayesian information criterion. The entropy of this distribution was obtained using power transformation technique. The properties of the special cases of this distribution for various values of parameters were discussed. 


\section{Double Weighted Distribution}

If two weight functions are used in the derivation of probability model, then the resultant distribution is known as double weighted distribution (DWD). The DWD can be obtained as

$$
f_{w}(x ; c)=\frac{w(x) K(x) K(c x)}{W D}, \quad x \geq 0, c>0
$$

where

$$
W D=\int \frac{w(x) K(x) K(c x)}{d} x \quad x, c>0 .
$$

and the first weight is $w(x)$ and the second weight is $K(c x)$. The concept of double weighted distribution was introduced by Al-Khadim and Hantoosh (2013) and later has been studied by other researchers.

Al-Kadim and Hantoosh (2013) presented double weighted exponential distribution (DWED) by considering the oneparameter exponential distribution with pdf

$$
f(x ; \lambda)=\lambda e^{(-\lambda x)}, \quad x, \lambda>0
$$

with the first weight function $w(x)=x$ and the second weight function $F(c x)=1-e^{(-c \lambda x)}$ in the equation (70). The derived double weighted exponential distribution has pdf

$$
f_{w}(x ; c, \lambda)=\frac{(c+1)^{2} \lambda^{2}}{(c+1)^{2}-1} x e^{-\lambda x}\left(1-e^{(-\lambda c x)}, \quad x, c, \lambda>0 .\right.
$$

Then, they derived the cdf as well as some other useful distributional properties of the new DWED. Method of moment and maximum likelihood were used to estimate the parameters of DWED. The calculations were illustrated with the help of a numerical example.

The properties and estimation of double weighted Rayleigh distribution (DWRD) were presented by Rashwan (2013). This paper developed DWRD by taking the first weight function $w(x)=x$ and the second $F(\alpha x)$, where $F(\alpha x)$ depend on the original distribution. Some statistical properties of this distribution were discussed. He also estimated the parameters of this distribution using the methods of moment and the maximum likelihood. The calculations were illustrated with the help of a numerical example.

Ahmad and Ahmad (2014) developed the characterization and estimation of DWRD. A double weighted inverse Weibull distribution (DWIWD) was obtainable by Al-Kadim and Hantoosh in (2014). They took $w(x)=x$, and $F(c x)$ as their first and second weight functions. Then, they derive some useful statistical properties of their distribution.

The modified double weighted exponential distribution (MDWED) and its properties were presented in Saghir et al.(2015). They employe $w(x)=e^{n x}$ and $F(c x)=1-e^{(-\lambda c x)}$ as their weight functions. The statistical properties of MDWED were explored. The Kolmogorov- Smirnov test was used to choose a better fitted probability model. The results of this test shown that MDWED is more suitable distribution to fit rainfall data than DWED.

\section{Characterization Results}

Our characterizations of the continuous weighted distributions are in terms of a simple relationship between two truncated moments. Our characterization results presented here will employ an interesting result due to Glänzel (1987) (Theorem 1 in Appendix A).

Remark 1. In Theorem 1, the interval $H$ need not be closed since the condition is only on the interior of $H$.

We will take up the distributions discussed in this section in the chronological order of appearances rather than their order of importance. In expressing these distributions we will use (as far as we can) the same symbols for the parameters as employed by the original authors. We shall use $f(\cdot)$ for $p d f$ (probability density function) and $F(\cdot)$ for its corresponding $c d f$ (cumulative distribution function). The advantage of this kind of characterization is that the $c d f$, $F(\cdot)$ does not have to have a closed form as long as its corresponding $p d f$ has a closed form.

1) TPWE (Gupta and Kundu, 2009):

Proposition 1. Let $X: \Omega \rightarrow(0, \infty)$ be a continuous random variable and let $q_{1}(x)=e^{\beta(1-\alpha) x}\left(1-e^{-\alpha \beta x}\right)^{-1}$ and $q_{2}(x)=e^{\beta(1-\alpha) x}$ for $x>0$. The random variable $X$ has $p d f$ (5) if and only if the function $\eta$ defined in Theorem1 has the form

$$
\eta(x)=\frac{1}{2}\left(2-e^{-\alpha \beta x}\right), \quad x>0 .
$$


Proof. Let $X$ be a random variable with $p d f(5)$, then

$$
(1-F(x)) E\left[q_{1}(x) \mid X \geq x\right]=\frac{\alpha+1}{\alpha^{2}} e^{-\alpha \beta x}, \quad x>0,
$$

and

and finally

$$
(1-F(x)) E\left[q_{2}(x) \mid X \geq x\right]=\frac{\alpha+1}{2 \alpha^{2}} e^{-\alpha \beta x}\left(2-e^{-\alpha \beta x}\right), \quad x>0,
$$

$$
\eta(x) q_{1}(x)-q_{2}(x)=\frac{q_{2}(x)}{2}\left(\frac{e^{-\alpha \beta x}}{1-e^{-\alpha \beta x}}\right)>0, \text { for } x>0 .
$$

Conversely, if $\eta$ is given as above, then

$$
s^{\prime}(x)=\frac{\eta^{\prime}(x) q_{1}(x)}{\eta(x) q_{1}(x)-q_{2}(x)}=\alpha \beta, \quad x>0,
$$

and hence

$$
s(x)=\alpha \beta x, \quad x>0 .
$$

Now, in view of Theorem1, $X$ has density (5).

Corollary 1. Let $X: \Omega \rightarrow(0, \infty)$ be a continuous random variable and let $q_{1}(x)$ be as in Proposition 1 . The $p d f$ of $X$ is (5) if and only if there exist functions $q_{2}$ and $\eta$ defined in Theorem 1 satisfying the differential equation

$$
\frac{\eta^{\prime}(x) q_{1}(x)}{\eta(x) q_{1}(x)-q_{2}(x)}=\alpha \beta, \quad x>0 .
$$

The general solution of the above differential equation is

$$
\eta(x)=e^{\alpha \beta x}\left[-\int \alpha \beta e^{-\alpha \beta x}\left(q_{1}(x)\right)^{-1} q_{2}(x) d x+D\right],
$$

where $D$ is a constant. Note that a set of functions satisfying the above differential equation is given in Proposition 1 with $D=0$. However, it should be also mentioned that there are other triplets $\left(q_{1}, q_{2}, \eta\right)$ satisfying the conditions of Theorem1.

Remark 2. Letting $q_{1}(x)=e^{\lambda(1-\alpha) x}$ and $q_{2}(x)=h(x)\left(1-e^{-\lambda \alpha x}\right)^{2}$ for $x>0$. The random variable $X$ has $p d f(5)$ if and only if the function $\eta$ defined in Theorem 1 has the form

$$
\eta(x)=\frac{1}{2}\left\{1+\left(1-e^{-\lambda \alpha x}\right)^{2}\right\}, \quad x>0 .
$$

The corresponding differential equation is

$$
\frac{\eta^{\prime}(x) q_{1}(x)}{\eta(x) q_{1}(x)-q_{2}(x)}=\frac{2 \lambda \alpha e^{-\lambda \alpha x}\left(1-e^{-\lambda \alpha x}\right)}{1-\left(1-e^{-\lambda \alpha x}\right)^{2}}, \quad x>0,
$$

and the general solution of the above differential equation is

$$
\eta(x)=\left[1-\left(1-e^{-\lambda \alpha x}\right)^{2}\right]^{-1}\left[-\int 2 \lambda \alpha e^{-\lambda \alpha x}\left(1-e^{-\lambda \alpha x}\right)\left(q_{1}(x)\right)^{-1} q_{2}(x) d x+D\right],
$$

where $D$ is a constant. Note that a set of functions satisfying the above differential equation is given in Remark 2 with $D=\frac{1}{2}$.

Remark 3. A Proposition and a Corollary similar to Proposition 1 and Corollary 1 will be stated (without proofs) for the remaining weighted distributions considered here.

2) WIWD (Kersey, 2010):

Proposition 2. Let $X: \Omega \rightarrow(0, \infty)$ be a continuous random variable and let $q_{1}(x)=e^{\beta(1-\alpha) x}\left(1-e^{-\alpha \beta x}\right)^{-1}$ and $q_{2}(x)=e^{\beta(1-\alpha) x}$ for $x>0$. The random variable $X$ has $p d f(7)$ if and only if the function $\eta$ defined in Theorem 1 has the form

$$
\eta(x)=\frac{1}{2}\left(1+e^{-(\alpha x)^{-\beta}}\right), \quad x>0 .
$$


Corollary 2. Let $X: \Omega \rightarrow(0, \infty)$ be a continuous random variable and let $q_{1}(x)$ be as in Proposition 2. The $p d f$ of $X$ is (7) if and only if there exist functions $q_{2}$ and $\eta$ defined in Theorem1 satisfying the differential equation

$$
\frac{\eta^{\prime}(x) q_{1}(x)}{\eta(x) q_{1}(x)-q_{2}(x)}=\frac{\alpha^{-\beta} \beta x^{-(\beta+1)} e^{-(\alpha x)^{-\beta}}}{1-e^{-(\alpha x)^{-\beta}}}, x>0 .
$$

The general solution of the above differential equation is

$$
\eta(x)=\left(1-e^{-(\alpha x)^{-\beta}}\right)^{-1}\left[-\int \alpha^{-\beta} \beta x^{-(\beta+1)} e^{-(\alpha x)^{-\beta}}\left(q_{1}(x)\right)^{-1} q_{2}(x) d x+D\right],
$$

where $D$ is a constant. Note that a set of functions satisfying the above differential equation is given in Proposition 2 with $D=\frac{1}{2}$.

3) Weibull (WBIW (Kersey, 2010):

Proposition 3. Let $X: \Omega \rightarrow(0, \infty)$ be a continuous random variable and let $q_{1}(x) \equiv 1$ and $q_{2}(x)=1-e^{-a(\alpha x)^{-\beta}}$ for $x>0$. The random variable $X$ has $p d f(10)$ if and only if the function $\eta$ defined in Theorem1 has the form

$$
\eta(x)=\frac{a}{a+1}\left(1-e^{-a(\alpha x)^{-\beta}}\right), \quad x>0 .
$$

Corollary 3. Let $X: \Omega \rightarrow(0, \infty)$ be a continuous random variable and let $q_{1}(x)$ be as in Proposition 3 . The $p d f$ of $X$ is (10) if and only if there exist functions $q_{2}$ and $\eta$ defined in Theorem 1 satisfying the differential equation

$$
\frac{\eta^{\prime}(x) q_{1}(x)}{\eta(x) q_{1}(x)-q_{2}(x)}=-\frac{a(a+1) \alpha^{-\beta} \beta x^{-(\beta+1)} e^{-a(\alpha x)^{-\beta}}}{1-e^{-a(\alpha x)^{-\beta}}}, x>0 .
$$

The general solution of the above differential equation is

$$
\eta(x)=\left(1-e^{-a(\alpha x)^{-\beta}}\right)^{-1}\left[\int a(a+1) \alpha^{-\beta} \beta x^{-(\beta+1)} e^{-a(\alpha x)^{-\beta}}\left(q_{1}(x)\right)^{-1} q_{2}(x) d x+D\right],
$$

where $D$ is a constant. Note that a set of functions satisfying the above differential equation is given in Proposition 3 with $D=0$.

4) WW (Roman, 2010):

Proposition 4. Let $X: \Omega \rightarrow(0, \infty)$ be a continuous random variable and let $q_{1}(x) \equiv 1$ and $q_{2}(x)=1-e^{-a(\alpha x)^{-\beta}}$ for $x>0$. The random variable $X$ has $p d f(13)$ if and only if the function $\eta$ defined in Theorem 1 has the form

$$
\eta(x)=\frac{a}{a+1}\left(1-e^{-a(\alpha x)^{-\beta}}\right), \quad x>0 .
$$

Corollary 4. Let $X: \Omega \rightarrow(0, \infty)$ be a continuous random variable and let $q_{1}(x)$ be as in Proposition 4 . The $p d f$ of $X$ is (13) if and only if there exist functions $q_{2}$ and $\eta$ defined in Theorem 1 satisfying the differential equation

$$
\frac{\eta^{\prime}(x) q_{1}(x)}{\eta(x) q_{1}(x)-q_{2}(x)}=\beta \theta^{-\beta} x^{\beta-1}, \quad x>0 .
$$

The general solution of the above differential equation is

$$
\eta(x)=e^{\left(\frac{x}{\theta} \beta^{\beta}\right.}\left[-\int \beta \theta^{-\beta} x^{\beta-1} e^{-\left(\frac{x}{\theta}\right)^{\beta}}\left(q_{1}(x)\right)^{-1} q_{2}(x) d x+D\right],
$$

where $D$ is a constant. Note that a set of functions satisfying the above differential equation is given in Proposition 4 with $D=0$.

5) WRD (Roman, 2010):

Proposition 5. Let $X: \Omega \rightarrow(0, \infty)$ be a continuous random variable and let $q_{1}(x)=x^{-c}$ and $q_{2}(x)=x^{-c} e^{-\frac{1}{2}\left(\frac{x}{\sigma}\right)^{2}}$ for $x>0$. The random variable $X$ has $p d f(15)$ if and only if the function $\eta$ defined in Theorem 1 has the form

$$
\eta(x)=\frac{1}{2} e^{-\frac{1}{2}\left(\frac{x}{\sigma}\right)^{2}}, \quad x>0 .
$$


Corollary 5. Let $X: \Omega \rightarrow(0, \infty)$ be a continuous random variable and let $q_{1}(x)$ be as in Proposition 5. The $p d f$ of $X$ is (15) if and only if there exist functions $q_{2}$ and $\eta$ defined in Theorem1 satisfying the differential equation

$$
\frac{\eta^{\prime}(x) q_{1}(x)}{\eta(x) q_{1}(x)-q_{2}(x)}=\frac{x}{\sigma^{2}}, x>0 .
$$

The general solution of the above differential equation is

$$
\eta(x)=e^{\frac{1}{2}\left(\frac{x}{\sigma}\right)^{2}}\left[-\int \frac{x}{\sigma^{2}}\left(q_{1}(x)\right)^{-1} q_{2}(x) d x+D\right],
$$

where $D$ is a constant. Note that a set of functions satisfying the above differential equation is given in Proposition 5 with $D=0$.

6) WGRD (Shi et al., 2012):

Proposition 6. Let $X: \Omega \rightarrow(0, \infty)$ be a continuous random variable and let $q_{1}(x)=x^{k-m-2}$ and $q_{2}(x)=q_{1}(x) e^{-\frac{x^{k}}{\theta}}$ for $x>0$. The random variable $X$ has $p d f(17)$ if and only if the function $\eta$ defined in Theorem 1 has the form

$$
\eta(x)=\frac{1}{2} e^{-\frac{k^{k}}{\theta}}, \quad x>0 .
$$

Corollary 6. Let $X: \Omega \rightarrow(0, \infty)$ be a continuous random variable and let $q_{1}(x)$ be as in Proposition 6 . The $p d f$ of $X$ is (17) if and only if there exist functions $q_{2}$ and $\eta$ defined in Theorem 1 satisfying the differential equation

$$
\frac{\eta^{\prime}(x) q_{1}(x)}{\eta(x) q_{1}(x)-q_{2}(x)}=\frac{k}{\theta} x^{k-1}, \quad x>0 .
$$

The general solution of the above differential equation is

$$
\eta(x)=e^{\frac{x^{k}}{\theta}}\left[-\int \frac{k}{\theta} x^{k-1} e^{-\frac{x^{k}}{\theta}}\left(q_{1}(x)\right)^{-1} q_{2}(x) d x+D\right],
$$

where $D$ is a constant. Note that a set of functions satisfying the above differential equation is given in Proposition 6 with $D=0$.

7) WGBD (Ye et al., 2012):

Proposition 7. Let $X: \Omega \rightarrow(0, \infty)$ be a continuous random variable and let $q_{1}(x)=x^{a(1-p)-k}\left[1+\left(\frac{x}{b}\right)^{a}\right]^{p+q-2}$ and $q_{2}(x)=q_{1}(x)\left[1+\left(\frac{x}{b}\right)^{a}\right]^{-1}$ for $x>0$. The random variable $X$ has $p d f(19)$ if and only if the function $\eta$ defined in Theorem1 has the form

$$
\eta(x)=\frac{1}{2}\left[1+\left(\frac{x}{b}\right)^{a}\right]^{-1}, \quad x>0 .
$$

Corollary 7. Let $X: \Omega \rightarrow(0, \infty)$ be a continuous random variable and let $q_{1}(x)$ be as in Proposition 7 . The $p d f$ of $X$ is (19) if and only if there exist functions $q_{2}$ and $\eta$ defined in Theorem 1 satisfying the differential equation

$$
\frac{\eta^{\prime}(x) q_{1}(x)}{\eta(x) q_{1}(x)-q_{2}(x)}=\frac{a x^{a-1}}{b^{a}\left[1+\left(\frac{x}{b}\right)^{a}\right]}, x>0 .
$$

The general solution of the above differential equation is

$$
\eta(x)=\left[1+\left(\frac{x}{b}\right)^{a}\right]\left[-\int \frac{a x^{a-1}}{b^{a}\left[1+\left(\frac{x}{b}\right)^{a}\right]^{2}}\left(q_{1}(x)\right)^{-1} q_{2}(x) d x+D\right],
$$

where $D$ is a constant. Note that a set of functions satisfying the above differential equation is given in Proposition 7 with $D=0$.

8) MWWD (Aleem et al., 2013):

Proposition 8. Let $X: \Omega \rightarrow(0, \infty)$ be a continuous random variable and let $q_{1}(x) \equiv 1$ and $q_{2}(x)=e^{-\beta\left(c \theta^{\gamma}+1\right) x^{\gamma}}$ for $x>0$. The random variable $X$ has $p d f(25)$ if and only if the function $\eta$ defined in Theorem1 has the form

$$
\eta(x)=\frac{1}{2} e^{-\beta\left(c \theta^{\gamma}+1\right) x^{\gamma}}, \quad x>0 .
$$


Corollary 8. Let $X: \Omega \rightarrow(0, \infty)$ be a continuous random variable and let $q_{1}(x)$ be as in Proposition 8 . The $p d f$ of $X$ is (25) if and only if there exist functions $q_{2}$ and $\eta$ defined in Theorem1 satisfying the differential equation

$$
\frac{\eta^{\prime}(x) q_{1}(x)}{\eta(x) q_{1}(x)-q_{2}(x)}=\beta \gamma\left(c \theta^{\gamma}+1\right) x^{\gamma-1}, x>0 .
$$

The general solution of the above differential equation is

$$
\eta(x)=e^{\beta\left(c \theta^{\gamma}+1\right) x^{\gamma}}\left[-\int \beta \gamma\left(c \theta^{\gamma}+1\right) x^{\gamma-1} e^{-\beta\left(c \theta^{\gamma}+1\right) x^{\gamma}}\left(q_{1}(x)\right)^{-1} q_{2}(x) d x+D\right],
$$

where $D$ is a constant. Note that a set of functions satisfying the above differential equation is given in Proposition 8 with $D=0$.

9) $\mathrm{EPWND}_{1}$ (Al-Khadim and Hantoosh, 2013):

Proposition 9. Let $X: \Omega \rightarrow \mathbb{R}$ be a continuous random variable and let $q_{1}(x)=(x-\mu) x^{-2}$ and $q_{2}(x)=q_{1}(x) e^{-\frac{(x-\mu)^{2}}{2 \sigma^{2}}}$ for $x \in \mathbb{R}$. The random variable $X$ has $p d f(26)$ if and only if the function $\eta$ defined in Theorem 1 has the form

$$
\eta(x)=\frac{1}{2} e^{-\frac{(x-\mu)^{2}}{2 \sigma^{2}}}, \quad x \in \mathbb{R} .
$$

Corollary 9. Let $X: \Omega \rightarrow \mathbb{R}$ be a continuous random variable and let $q_{1}(x)$ be as in Proposition 9. The $p d f$ of $X$ is (26) if and only if there exist functions $q_{2}$ and $\eta$ defined in Theorem 1 satisfying the differential equation

$$
\frac{\eta^{\prime}(x) q_{1}(x)}{\eta(x) q_{1}(x)-q_{2}(x)}=\frac{x-\mu}{\sigma^{2}}, \quad x \in \mathbb{R} .
$$

The general solution of the above differential equation is

$$
\eta(x)=e^{\frac{(x-\mu)^{2}}{2 \sigma^{2}}}\left[-\int \frac{(x-\mu)}{\sigma^{2}} e^{-\frac{(x-\mu)^{2}}{2 \sigma^{2}}}\left(q_{1}(x)\right)^{-1} q_{2}(x) d x+D\right],
$$

where $D$ is a constant. Note that a set of functions satisfying the above differential equation is given in Proposition 9 with $D=0$.

10) $\mathrm{EPWND}_{2}$ (Al-Khadim and Hantoosh, 2013):

Proposition 10. Let $X: \Omega \rightarrow \mathbb{R}$ be a continuous random variable and let $q_{1}(x)=(x-\mu) e^{-2 r x}$ and $q_{2}(x)=q_{1}(x) e^{-\frac{(x-\mu)^{2}}{2 \sigma^{2}}}$ for $x \in \mathbb{R}$. The random variable $X$ has $p d f(27)$ if and only if the function $\eta$ defined in Theorem 1 has the form

$$
\eta(x)=\frac{1}{2} e^{-\frac{(x-\mu)^{2}}{2 \sigma^{2}}}, \quad x \in \mathbb{R} .
$$

Corollary 10. Let $X: \Omega \rightarrow \mathbb{R}$ be a continuous random variable and let $q_{1}(x)$ be as in Proposition 10. The $p d f$ of $X$ is (27) if and only if there exist functions $q_{2}$ and $\eta$ defined in Theorem 1 satisfying the differential equation

$$
\frac{\eta^{\prime}(x) q_{1}(x)}{\eta(x) q_{1}(x)-q_{2}(x)}=\frac{x-\mu}{\sigma^{2}}, \quad x \in \mathbb{R} .
$$

The general solution of the above differential equation is

$$
\eta(x)=e^{\frac{(x-\mu)^{2}}{2 \sigma^{2}}}\left[-\int \frac{(x-\mu)}{\sigma^{2}} e^{-\frac{(x-\mu)^{2}}{2 \sigma^{2}}}\left(q_{1}(x)\right)^{-1} q_{2}(x) d x+D\right],
$$

where $D$ is a constant. Note that a set of functions satisfying the above differential equation is given in Proposition 10 with $D=0$.

11) WWD (Mahdy, 2013):

Proposition 11. Let $X: \Omega \rightarrow(0, \infty)$ be a continuous random variable and let $q_{1}(x)=\left(1-e^{-\lambda(\alpha x)^{\beta}}\right)^{-1}$ and $q_{2}(x)=$ $q_{1}(x) e^{-\lambda x^{\beta}}$ for $x>0$. The random variable $X$ has $p d f(28)$ if and only if the function $\eta$ defined in Theorem1 has the form

$$
\eta(x)=\frac{1}{2} e^{-\lambda x^{\beta}}, \quad x>0 .
$$


Corollary 11. Let $X: \Omega \rightarrow(0, \infty)$ be a continuous random variable and let $q_{1}(x)$ be as in Proposition 11 . The $p d f$ of $X$ is (28) if and only if there exist functions $q_{2}$ and $\eta$ defined in Theorem1 satisfying the differential equation

$$
\frac{\eta^{\prime}(x) q_{1}(x)}{\eta(x) q_{1}(x)-q_{2}(x)}=\lambda \beta x^{\beta-1}, \quad x>0 .
$$

The general solution of the above differential equation is

$$
\eta(x)=e^{\lambda x^{\beta}}\left[-\int \lambda \beta x^{\beta-1} e^{-\lambda x^{\beta}}\left(q_{1}(x)\right)^{-1} q_{2}(x) d x+D\right],
$$

where $D$ is a constant. Note that a set of functions satisfying the above differential equation is given in Proposition 11 with $D=0$.

12) EWWD (Idowu and Adebayo, 2014):

Proposition 12. Let $X: \Omega \rightarrow(0, \infty)$ be a continuous random variable and let $q_{1}(x)=\left(1-e^{-\lambda x^{\beta}}\right)$ and $q_{2}(x) \equiv 1$ for $x>0$. The random variable $X$ has $p d f(29)$ if and only if the function $\eta$ defined in Theorem 1 has the form

$$
\eta(x)=1-\frac{1}{2} e^{-\lambda x^{\beta}}, \quad x>0 .
$$

Corollary 12. Let $X: \Omega \rightarrow(0, \infty)$ be a continuous random variable and let $q_{1}(x)$ be as in Proposition 12. The $p d f$ of $X$ is (29) if and only if there exist functions $q_{2}$ and $\eta$ defined in Theorem 1 satisfying the differential equation

$$
\frac{\eta^{\prime}(x) q_{1}(x)}{\eta(x) q_{1}(x)-q_{2}(x)}=\lambda \beta x^{\beta-1}, \quad x>0 .
$$

The general solution of the above differential equation is

$$
\eta(x)=e^{\lambda x^{\beta}}\left[-\int \lambda \beta x^{\beta-1} e^{-\lambda x^{\beta}}\left(q_{1}(x)\right)^{-1} q_{2}(x) d x+D\right],
$$

where $D$ is a constant. Note that a set of functions satisfying the above differential equation is given in Proposition 12 with $D=0$.

13) WFPD (Odubote and Oluyede, 2014):

Proposition 13. Let $X: \Omega \rightarrow(\mu, \infty)$ be a continuous random variable and let $q_{1}(x)=\left(\frac{x-\mu}{\theta}\right)^{\frac{1-\beta}{\gamma}-k}\left(1+\left(\frac{x-\mu}{\theta}\right)^{\frac{1}{\gamma}}\right)^{-1}$ and $q_{2}(x)=\left(\frac{x-\mu}{\theta}\right)^{\frac{1-\beta}{\gamma}-k}\left(1+\left(\frac{x-\mu}{\theta}\right)^{\frac{1}{\gamma}}\right)^{-2}$ for $x>\mu$. The random variable $X$ has $p d f(31)$ if and only if the function $\eta$ defined in Theorem1 has the form

$$
\eta(x)=\frac{\alpha+\beta}{\alpha+\beta+1}\left(1+\left(\frac{x-\mu}{\theta}\right)^{\frac{1}{\gamma}}\right)^{-1}, \quad x>\mu .
$$

Corollary 13. Let $X: \Omega \rightarrow(\mu, \infty)$ be a continuous random variable and let $q_{1}(x)$ be as in Proposition 13. The $p d f$ of $X$ is (31) if and only if there exist functions $q_{2}$ and $\eta$ defined in Theorem 1 satisfying the differential equation

$$
\frac{\eta^{\prime}(x) q_{1}(x)}{\eta(x) q_{1}(x)-q_{2}(x)}=\frac{(\alpha+\beta)\left(\frac{x-\mu}{\theta}\right)^{\frac{1}{\gamma}}}{\gamma \theta\left(1+\left(\frac{x-\mu}{\theta}\right)^{\frac{1}{\gamma}}\right)}, \quad x>\mu .
$$

The general solution of the above differential equation is

$$
\eta(x)=\left(1+\left(\frac{x-\mu}{\theta}\right)^{\frac{1}{\gamma}}\right)\left[-\int(\alpha+\beta)\left(\frac{x-\mu}{\theta}\right)^{\frac{1}{\gamma}}\left(1+\left(\frac{x-\mu}{\theta}\right)^{\frac{1}{\gamma}}\right)^{-1}\left(q_{1}(x)\right)^{-1} q_{2}(x) d x+D\right],
$$

where $D$ is a constant. Note that a set of functions satisfying the above differential equation is given in Proposition 13 with $D=0$.

14) WLD (Bashir and Rasul, 2015): 
Proposition 14. Let $X: \Omega \rightarrow(0, \infty)$ be a continuous random variable and let $q_{1}(x)=(1+x)^{-1}$ and $q_{2}(x)=$ $(1+x)^{-1} e^{-x(\theta-\phi)}$ for $x>0$. Then, the random variable $X$ has $p d f$ (33) if and only if the function $\eta$ defined in Theorem1 has the form

$$
\eta(x)=\frac{1}{2} e^{-x(\theta-\phi)}, \quad x>0 .
$$

Corollary 14. Let $X: \Omega \rightarrow(0, \infty)$ be a continuous random variable and let $q_{1}(x)$ be as in Proposition 14 . The $p d f$ of $X$ is (33), if and only if there exist functions $q_{2}$ and $\eta$ defined in Theorem 1 satisfying the differential equation

$$
\frac{\eta^{\prime}(x) q_{1}(x)}{\eta(x) q_{1}(x)-q_{2}(x)}=\theta-\phi, \quad x>0 .
$$

The general solution of the above differential equation is

$$
\eta(x)=e^{x(\theta-\phi)}\left[-\int(\theta-\phi) e^{-x(\theta-\phi)}\left(q_{1}(x)\right)^{-1} q_{2}(x) d x+D\right],
$$

where $D$ is a constant. Note that a set of functions satisfying the above differential equation is given in Proposition 14 with $D=0$.

15) WGED (Mahdavi, 2015):

Proposition 15. Let $X: \Omega \rightarrow(0, \infty)$ be a continuous random variable and let $q_{1}(x)=\left(1-e^{-\alpha \lambda x}\right)^{-1}$ and $q_{2}(x)=$ $h(x)\left(1-e^{-\lambda x}\right)^{\beta}$ for $x>0$. The random variable $X$ has $p d f(35)$ if and only if the function $\eta$ defined in Theorem 1 has the form

$$
\eta(x)=\frac{1}{2}\left\{1+\left(1-e^{-\lambda x}\right)^{\beta}\right\}, \quad x>0 .
$$

Corollary 15. Let $X: \Omega \rightarrow(0, \infty)$ be a continuous random variable and let $q_{1}(x)$ be as in Proposition 15 . The $p d f$ of $X$ is (35) if and only if there exist functions $q_{2}$ and $\eta$ defined in Theorem 1 satisfying the differential equation

$$
\frac{\eta^{\prime}(x) q_{1}(x)}{\eta(x) q_{1}(x)-q_{2}(x)}=\frac{\lambda \beta e^{-\lambda x}\left(1-e^{-\lambda x}\right)^{\beta-1}}{1-\left(1-e^{-\lambda x}\right)^{\beta}}, \quad x>0 .
$$

The general solution of the above differential equation is

$$
\eta(x)=\left[1-\left(1-e^{-\lambda x}\right)^{\beta}\right]^{-1}\left[-\int \lambda \beta e^{-\lambda x}\left(1-e^{-\lambda x}\right)^{\beta-1}\left(q_{1}(x)\right)^{-1} q_{2}(x) d x+D\right],
$$

where $D$ is a constant. Note that a set of functions satisfying the above differential equation is given in Proposition 15 with $D=\frac{1}{2}$.

16) WWD (Nasiru, 2015):

Proposition 16. Let $X: \Omega \rightarrow(0, \infty)$ be a continuous random variable and let $q_{1}(x)=e^{-\alpha(\lambda)^{\theta}}$ and $q_{2}(x)=h(x) e^{-\alpha x^{\theta}}$ for $x>0$. The random variable $X$ has $p d f(37)$ if and only if the function $\eta$ defined in Theorem1 has the form

$$
\eta(x)=\frac{1}{2} e^{-\alpha x^{\theta}}, \quad x>0 .
$$

Corollary 16. Let $X: \Omega \rightarrow(0, \infty)$ be a continuous random variable and let $q_{1}(x)$ be as in Proposition 16 . The $p d f$ of $X$ is (37) if and only if there exist functions $q_{2}$ and $\eta$ defined in Theorem 1 satisfying the differential equation

$$
\frac{\eta^{\prime}(x) q_{1}(x)}{\eta(x) q_{1}(x)-q_{2}(x)}=\alpha \theta x^{\theta-1}, \quad x>0 .
$$

The general solution of the above differential equation is

$$
\eta(x)=e^{\alpha x^{\theta}}\left[-\int \alpha \theta x^{\theta-1} e^{-\alpha x^{\theta}}\left(q_{1}(x)\right)^{-1} q_{2}(x) d x+D\right],
$$

where $D$ is a constant. Note that a set of functions satisfying the above differential equation is given in Proposition 16 with $D=0$.

17) WGED (Rezzoky and Nadhel, 2015): 
Proposition 17. Let $X: \Omega \rightarrow(0, \infty)$ be a continuous random variable and let $q_{1}(x)=x^{-1}$ and $q_{2}(x)=x^{-1}\left(1-e^{-\lambda x}\right)^{2}$ for $x>0$. The random variable $X$ has $p d f$ (39) if and only if the function $\eta$ defined in Theorem 1 has the form

$$
\eta(x)=\frac{1}{2}\left\{1+\left(1-e^{-\lambda x}\right)^{2}\right\}, \quad x>0 .
$$

Corollary 17. Let $X: \Omega \rightarrow(0, \infty)$ be a continuous random variable and let $q_{1}(x)$ be as in Proposition 17. The $p d f$ of $X$ is (39) if and only if there exist functions $q_{2}$ and $\eta$ defined in Theorem 1 satisfying the differential equation

$$
\frac{\eta^{\prime}(x) q_{1}(x)}{\eta(x) q_{1}(x)-q_{2}(x)}=\frac{2 \lambda e^{-\lambda x}\left(1-e^{-\lambda x}\right)}{1-\left(1-e^{-\lambda x}\right)^{2}}, \quad x>0 .
$$

The general solution of the above differential equation is

$$
\eta(x)=\left\{1-\left(1-e^{-\lambda x}\right)^{2}\right\}^{-1}\left[-\int 2 \lambda e^{-\lambda x}\left(1-e^{-\lambda x}\right)\left(q_{1}(x)\right)^{-1} q_{2}(x) d x+D\right],
$$

where $D$ is a constant. Note that a set of functions satisfying the above differential equation is given in Proposition 17 with $D=\frac{1}{2}$.

18) EWED (Oguntunde, 2015):

Proposition 18. Let $X: \Omega \rightarrow(0, \infty)$ be a continuous random variable and let $q_{1}(x)=e^{(1-\alpha) \lambda x}\left[1-e^{-\lambda x}-\frac{1}{1+\alpha}\left(1-e^{-(1+\alpha) \lambda x}\right)\right]^{1-\theta}$ and $q_{2}(x)=h(x)\left(1-e^{-\lambda \alpha x}\right)^{2}$ for $x>0$. The random variable $X$ has $p d f(43)$ if and only if the function $\eta$ defined in Theorem1 has the form

$$
\eta(x)=\frac{1}{2}\left\{1+\left(1-e^{-\lambda \alpha x}\right)^{2}\right\}, \quad x>0 .
$$

Corollary 18. Let $X: \Omega \rightarrow(0, \infty)$ be a continuous random variable and let $q_{1}(x)$ be as in Proposition 18 . The $p d f$ of $X$ is (43) if and only if there exist functions $q_{2}$ and $\eta$ defined in Theorem 1 satisfying the differential equation

$$
\frac{\eta^{\prime}(x) q_{1}(x)}{\eta(x) q_{1}(x)-q_{2}(x)}=\frac{2 \lambda \alpha e^{-\lambda \alpha x}\left(1-e^{-\lambda \alpha x}\right)}{1-\left(1-e^{-\lambda \alpha x}\right)^{2}}, \quad x>0 .
$$

The general solution of the above differential equation is

$$
\eta(x)=\left\{1-\left(1-e^{-\lambda \alpha x}\right)^{2}\right\}^{-1}\left[-\int 2 \lambda \alpha e^{-\lambda \alpha x}\left(1-e^{-\lambda \alpha x}\right)\left(q_{1}(x)\right)^{-1} q_{2}(x) d x+D\right],
$$

where $D$ is a constant. Note that a set of functions satisfying the above differential equation is given in Proposition 18 with $D=\frac{1}{2}$.

19) NWLD (Asgharzadeh et al., 2016):

Proposition 19. Let $X: \Omega \rightarrow(0, \infty)$ be a continuous random variable and let $q_{1}(x)=(1+x)^{-1} e^{(1-\alpha) \lambda x}$ and $q_{2}(x)=$ $h(x)\left(1-e^{-\lambda \alpha x}\right)^{2}$ for $x>0$. The random variable $X$ has $p d f(44)$ if and only if the function $\eta$ defined in Theorem1 has the form

$$
\eta(x)=\frac{1}{2}\left\{1+\left(1-e^{-\lambda \alpha x}\right)^{2}\right\}, \quad x>0 .
$$

Corollary 18. Let $X: \Omega \rightarrow(0, \infty)$ be a continuous random variable and let $q_{1}(x)$ be as in Proposition 19. The $p d f$ of $X$ is (44) if and only if there exist functions $q_{2}$ and $\eta$ defined in Theorem 1 satisfying the differential equation

$$
\frac{\eta^{\prime}(x) q_{1}(x)}{\eta(x) q_{1}(x)-q_{2}(x)}=\frac{2 \lambda \alpha e^{-\lambda \alpha x}\left(1-e^{-\lambda \alpha x}\right)}{1-\left(1-e^{-\lambda \alpha x}\right)^{2}}, \quad x>0 .
$$

The general solution of the above differential equation is

$$
\eta(x)=\left\{1-\left(1-e^{-\lambda \alpha x}\right)^{2}\right\}^{-1}\left[-\int 2 \lambda \alpha e^{-\lambda \alpha x}\left(1-e^{-\lambda \alpha x}\right)\left(q_{1}(x)\right)^{-1} q_{2}(x) d x+D\right],
$$

where $D$ is a constant. Note that a set of functions satisfying the above differential equation is given in Proposition 19 with $D=\frac{1}{2}$. 


\section{Concluding Remarks}

A brief review has been presented on weighted distributions theory and applications. The weighted distributions seem to occur frequently in various fields of real life. The weighted distribution theory gives a unified approach to modeling the biased data and should be taught in probability and mathematical statistics courses. The construction, study and applications of weighted distributions and their other related categories, is one of the classical and active fields of research in probability and mathematical statistics. It provides consolidating formulation for correction of biases that exist in unequally weighted sample data. Also, the theory provides a means of fitting models to the unknown weighting function when samples can be taken both from the original distribution and the resulting 'biased' distribution. These problems exist in all disciplines of science and numerous ad hoc solutions have been developed. In recent years several research papers containing theory about the weighted distributions have been published that we have discussed in this article. For instance, a detailed study on the construction of various weighted distributions by numerous authors, are confabulated. Due to the fact that this area is such an active field of research, new models are constantly being discovered. What follows is a list of further weighted models with the directions given that could be carried out.

- Length - biased and length - biased weighted versions of Poisson distribution can be developed for modeling of counts data, no studies, as far as we gathered, have been carried out in this area.

- Length - biased and length - biased weighted cases of the generalized beta distribution of the second kind can be developed.

- For efficient modeling of the carbon data, the length - biased and weighted classes of the Lindley distribution can be formulated.

- Weighted lognormal distribution and length - biased weighted lognormal distribution can be developed for analysis of data from oil field explorations.

- We can attain the weighted and length - biased weighted versions of the beta- Pareto distribution for the modeling of Norwegian fire claims data set.

- Double weighted Weibull distribution can be developed for modeling of revolutions to failure for 23 ball bearings in fatigue test data set.

- Double weighted version of the exponentiated inverted Weibull distribution can be accomplished for the modeling of uncensored data on distance between cracks in a pipe data set.

- Weighted edition of Rayleigh distribution can be obtainable for the modeling of real data set corresponds to the exceedances of flood peaks (in $\mathrm{m} 3 / \mathrm{s}$ ) of the Wheaton River near Carcross in Youkon territory, Canada, etc.

\section{References}

Adhikari, M. T. R., \& Srivastava, R. S. (2014). Poisson-Size-biased Lindley Distribution. International Journal of Science and Research Publications, 4, 2250-3153.

Ahmad, A., \& Ahmad, S. P. (2014). Characterization and Estimation of Double Weighted Rayleigh Distribution. Journal of Agriculture and Life Science, 1, 2375-4214.

Ahmad, A., Reshi, J. A., \& Mir, K. A. (2013). Structural properties of size biased Gamma distribution. Journal of Mathematics, 5, 55-61.

Aleem, M., Sufyan, M., \& Khan, N. S. (2013). A Class of Modified Weighted Weibull Distribution and its properties. American Review of Mathematics and Statistics, 1, 29-37.

Al-Kadim, K. A., \& Hantoosh, A. F. (2013). Even-Power Weighted Distribution. Mathematical Theory and Modeling, 3, 40-48.

Al-Kadim, K. A., \& Hantoosh, A. F. (2014). Double Weighted Inverse Weibull Distribution. Proceedings Book of ICETSR, Malaysia, 386-398.

Al-Kadim, K., \& Hantoosh, A. F. (2013). Double Weighted Distribution and Double Weighted Exponential Distribution. Mathematical Theory and Modeling, 3, 124-34.

Al-Khadim, A. K., \& Hussein, A. N. (2014). New proposed length biased weighted Exponential and Rayleigh distribution with application. Mathematical Theory and Modeling, 4(7), 137-52. 
Alqallaf, F., Ghitany, M. E., \& Agostinelli, C. (2015). Weighted Exponential distribution: Different methods of Estimations. Applied Mathematical and Information Science, 9,1167-73.

Azzalini, A., 1985. A class of distribution which includes the normal ones. Scandinavian Journal of Statistics, 12, 171-178.

Badmus, N. I., Bamiduro, T. A., \& Ogunobi, S. G. (2014). Lehmann Type-II Weighted Weibull distribution. International Journal Physical Science, 9, 71-78.

Bashir, S., \& Ahmad, M. (2014). Record Values from Size-Biased Pareto Distribution and a Characterization. International Journal of Engineering Research, 2, 101-109.

Bashir, S., \& Akhtar, K. (2014). Record Values on The Size-Biased Student's t Distribution. International Journal Innovative Science Engineering and Technology, 1, 344-348.

Bashir, S., \& Rasul, M. (2015). Some Properties of the Weighted Lindley distribution. International Journal of Economics and Business Review, 3, 11-17.

Castillo, J. D., \& Casany, M. P. (1998). Weighted Poison Distribution for over dispersion and under dispersion Solutions. Annals of Institute of Statistical Mathematics, 50, 567-585.

Das, K. K; \& Roy, T. D.(2011a). Applicability of Length Biased Generalized Rayleigh distribution. Advances in Applied Science Research, 2, 320-327.

Das, K. K., \& Roy, T. D. (2011b). On Some Length-Biased Weighted Weibull Distribution. Advances in Applied Science Research, 2, 465-475.

Dey, S., Dey, T., \& Anis, M. Z. (2015). Weighted Weibull distribution: Properties and Estimation. Journal of Statistical Theory and Practice, 9, 250-265.

Domma, F., Condino. F., \& Popovic, B. V. (2016). A new generalized weighted Weibull distribution with decreasing, increasing, upside-down bathtub,N-shape and M-shape hazard rate. Journal of Applied Statistics, http://dx.doi.org/ 10.1080/02664763.2016.1267118.

Fay, D., Haddadi, H., Uhlig, S., Moore, A. W., Mortier, R., \& Jamakovic, A. (2008). Weighted spectral distribution. Technical Report, UCAM-CL-TR-729, 1476-2986.

Fisher, R. A. (1934). The effects of methods of ascertainment upon the estimation of frequencies. The Annals of Eugenics, 6, 13-25.

Ghitany M. E., Al-Mutairi D. K., \& Husain, H. A. (2011). A two-parameter weighted Lindley distribution and its applications to survival data. Mathematics and Computers in Simulation, 81, 1190-1201.

Ghitany, M. E., \& Al-Mutairi, D. K. (2008). Size-biased Poisson-Lindley distribution and its application. International Journal of Statistics, 3, 299-311.

Glänzel, W., A characterization theorem based on truncated moments and its application to some distribution families, Mathematical Statistics and Probability Theory (Bad Tatzmannsdorf, 1986), Vol. B, Reidel, Dordrecht, 1987, 75-84.

Glänzel, W., Some consequences of a characterization theorem based on truncated moments, Statistics: A Journal of Theoretical and Applied Statistics, 21 (4), 1990, 613-618.

Gupta, R. C., \& Akman, H. O.(1998). On the reliability studies of a weighted inverse Gaussian model. Journal of Statistical Planning and Inference, 48, 69-83.

Gupta, R. C., \& Keating, J. P. (1985). Relations for Reliability Measures under Length Biased Sampling. Scandinavian Journal of Statistics, 13, 49-56.

Gupta, R. C., \& Kirmani, S. N. (1990). The role of weighted distributions in stochastic modeling. Communication in Statistics - Theory and Methods, 19, 3147-3162.

Gupta, R. D., \& Kundu, D. A. (2009). New class of weighted exponential distribution. Statistics, 43, 621-634.

Hassan, A., \& Ahmad, P. B. (2009). Misclassified Size-Biased Modified Power Series Distribution and Its Applications. Mathematica Bohemica, 1, 1-17.

Idowu, B. N., \& Adebayo, B. T. (2014). Some Statistical Properties of Exponentiated Weighted Weibull Distribution. Global Journal of Science Frontier Research, 14, 2249-4626.

Jabeen, S., \& Jan, T. R. (2015). Information Measures of Size Biased Generalized Gamma Distribution. International 
Journal of Scientific Engineering and Applied Science, 1, 2395-3470.

Jones, M.C. (2004). Families of distributions arising from distributions of order statistics. Test, 13, 1-43.

Kersey, J. X. (2010). Weighted Inverse Weibull and Beta-Inverse Weibull distribution. M.SC.Thesis, university of Georgia Southern.

Kersey, J., \& Oluyede, B. O. (2012). Theoretical Properties of the Length-Biased Inverse Weibull distribution. Involve Journal of mathematics, 5, 379-391.

Khatree, R. (1989). Characterization of Inverse-Gaussian and Gamma distributions through their length biased distribution. IEEE Transactions on Reliability, 38, 610-611.

Mahdavi, A. (2015). Two Weighted Distributions Generated by Exponential Distribution. Journal of Mathematical Extension, 9, 1-12.

Mahday, A. (2013). A class of Weighted Weibull distributions and its properties. Studies in Mathematical Science, 6, 35-45.

Mir, K. A. (2009a). A New Method of Estimation of Size-Biased Generalized Logarithmic Series distribution.The Open Statistics and Probability Journal, 1, 20-24.

Mir, K. A. (2009b). On Size-Biased Negative Binomial Distribution and its Use in Zero-Truncated Cases. Measurement Science Review, 9, 33-35.

Mir, K., Ahmed, A., \& Reshi, J.(2013). Structural properties of length biased beta distribution of first kind. American Journal of Engineering Research, 2, 01-06.

Mizaal, A. R. (2015). Mathematical Study of Weighted Two Parameters Exponential Distribution. Magistra, 93, 8-18.

Modi, K. (2015). Length-Biased Weighted Maxwell Distribution. Pakistan Journal of Statistics and operation research, $11,465-472$.

Mudasir, S., \& Ahmad, S. P. (2015). Structural Properties of Length-Biased Nakagami Distribution. International Journal of Modern Mathematical Sciences, 13, 217-227.

Nasiru, S.(2015). Another Weighted Weibull Distribution from Azzalini’s Family. European Scientific Journal, 11, 134144.

Odubote, O., \& Oluyede, B. O. (2014). Theoretical properties of the weighted Feller-Pareto and Related Distributions. Asian Journal of Mathematics and Applications, 0173, 1-12.

Oguntunde, P. E. (2015). On the Exponentiated Weighted Exponential Distribution and Its Basic Statistical Properties. Applied Science Reports, 10, 160-167.

Oluyede, B. O. (1999). On Inequalities and Selection of Experiments for Length-Biased Distributions. Probability in the Engineering and Informational Sciences, 13, 169-185.

Patil, G. P., \& Rao, G. R. (1978). Weighted distributions and size biased sampling with applications to wildlife populations and human families. Biometrics, 34, 179-189.

Patil, G.P., Ord, J. K., Armitage, P.,\& Colton, T. (1997). Weighted distributions, in Encyclopaedia of Bio-statistics, 6th ed, Wiley, Chichester.

Rao, C. R. (1965). On discrete distributions arising out of methods of ascertainment, in Classical and Contagious Discrete Distribution. Patil, G. P. ed. Pergamon Press and Statistical Publishing Society, Calcutta, 320-332.

Rashwan, N. I. (2013). The Double Weighted Rayleigh Distribution Properties and Estimation. International Journal of Scientific and Engineering Research, 4, 1084-1089.

Ratnaparkhi, M. V., \& Naik-Nimbalkar, U. V. (2012). The Length-biased Lognormal Distribution and its application in the Analysis of data from oil Field Exploration studies. Modern Applied Statistical Methods, 11, 225-260.

Roman, R. (2010). Theoretical Properties and Estimation in Weighted Weibull and Related Distributions. Electronic Theses and Dissertations.

Saghir A., Saleem, M., Khadim, A., \& Tazeem, S. (2015). The Modified Double Weighted Exponential Distribution with Properties. Mathematical Theory and Modeling, 5, 2224-5804.

Seenoi, P., Supapa, K. T., \& Bodhisuwan, W. (2014). The Length Biased Exponentiated Inverted Weibull Distribution. International Journal of Pure and Applied Mathematics, 92,191-206. 
Shakhatreh, M. K. (2012). A two-parameter of Weighted exponential distributions. Statistics and Probability Letters, 82 , 252-261.

Sherina, V., \& Oluyede, B. O. (2014). Weighted Inverse Weibull Distribution: Statistical Properties and Applications. Theoretical Mathematics and Applications, 4, 1-30.

Shi, X., Broderick, O., \& Pararai, M. (2012). Theoretical Properties of Weighted Generalized Rayleigh and Related Distributions. Theoretical Mathematics and Applications, 2, 45-62.

Sing, K. L., \& Srivastava, R. S. (2014). Estimation of the Parameter in the Size-Biased Inverse Maxwell Distribution. International Journal of Statistica and Mathematica, 10, 52-56.

Ye, Y., Oluyede, B. O., \& Pararai, M. (2012). Weighted Generalized Beta Distribution of the Second Kind and Related Distributions. Journal of Statistical and Econometric Methods, 1, 13-31.

\section{Appendix A}

Theorem 1. Let $(\Omega, \mathcal{F}, \mathbf{P})$ be a given probability space and let $H=[a, b]$ be an interval for some $a<b$ $(a=-\infty, b=\infty$ might as well be allowed). Let $X: \Omega \rightarrow H$ be a continuous random variable with the distribution function $F$ and let $q_{1}$ and $q_{2}$ be two real functions defined on $H$ such that

$$
\mathbf{E}\left[q_{2}(X) \mid X \geq x\right]=\mathbf{E}\left[q_{1}(X) \mid X \geq x\right] \eta(x), \quad x \in H,
$$

is defined with some real function $\eta$. Assume that $q_{1}, q_{2}$ are continuous functions, $\eta$ has continuous derivative and $F$ is twice continuously differentiable and strictly monotone function on the set $H$. Finally, assume that the equation $\eta q_{1}=q_{2}$ has no real solution in the interior of $H$. Then $F$ is uniquely determined by the functions $q_{1}, q_{2}$ and $\eta$, particularly

$$
F(x)=\int_{a}^{x} C\left|\frac{\eta^{\prime}(u)}{\eta(u) q_{1}(u)-q_{2}(u)}\right| \exp (-s(u)) d u,
$$

where the function $s$ is a solution of the differential equation $s^{\prime}=\frac{\eta^{\prime} q_{1}}{\eta q_{1}-q_{2}}$ and $C$ is a constant, chosen to make $\int_{H} d F=1$.

We like to mention that this kind of characterization based on the ratio of truncated moments is stable in the sense of weak convergence (see Glänzel (1990)), in particular, let us assume that there is a sequence $\left\{X_{n}\right\}$ of random variables with distribution functions $\left\{F_{n}\right\}$ such that the functions $q_{1, n}, q_{2, n}$ and $\eta_{n}(n \in \mathbb{N})$ satisfy the conditions of Theorem 1 and let $q_{1, n} \rightarrow q_{1}, q_{2, n} \rightarrow q_{2}$ for some continuously differentiable real functions $q_{1}$ and $q_{2}$. Let, finally, $X$ be a random variable with distribution $F$. Under the condition that $q_{1, n}(X)$ and $q_{2, n}(X)$ are uniformly integrable and the family $\left\{F_{n}\right\}$ is relatively compact, the sequence $X_{n}$ converges to $X$ in distribution if and only if $\eta_{n}$ converges to $\eta$, where

$$
\eta(x)=\frac{E\left[q_{2}(X) \mid X \geq x\right]}{E\left[q_{1}(X) \mid X \geq x\right]} .
$$

This stability theorem makes sure that the convergence of distribution functions is reflected by corresponding convergence of the functions $q_{1}, q_{2}$ and $\eta$, respectively. It guarantees, for instance, the 'convergence' of characterization of the Wald distribution to that of the Lévy-Smirnov distribution if $\alpha \rightarrow \infty$.

A further consequence of the stability property of Theorem 1 is the application of this theorem to special tasks in statistical practice such as the estimation of the parameters of discrete distributions. For such purpose, the functions $q_{1}, q_{2}$ and, specially, $\eta$ should be as simple as possible. Since the function triplet is not uniquely determined it is often possible to choose $\eta$ as a linear function. Therefore, it is worth analyzing some special cases which helps to find new characterizations reflecting the relationship between individual continuous univariate distributions and appropriate in other areas of statistics.

\section{Copyrights}

Copyright for this article is retained by the author(s), with first publication rights granted to the journal.

This is an open-access article distributed under the terms and conditions of the Creative Commons Attribution license (http://creativecommons.org/licenses/by/4.0/). 